\title{
Barrier-Mediated Pulsatile Release
}

\author{
By Swapnil Gandhi ${ }^{a}$, Matthew D. Gosse ${ }^{a}$, Yasuhiro Nishii ${ }^{a, b}$, and Eric Nuxoll ${ }^{a, *}$ \\ ${ }^{a}$ Department of Chemical and Biochemical Engineering \\ 4133 Seamans Center for the Engineering Arts \& Sciences \\ University of Iowa \\ Iowa City, IA 52242, U.S.A. \\ ${ }^{\mathrm{b}}$ Department of Applied Chemistry and Biotechnology \\ Niihama National College of Technology \\ Ehime, 792-8580, Japan \\ *corresponding author \\ email: eric-nuxoll@uiowa.edu \\ office: 1-319-353-2377 \\ fax: 1-319-335-1415
}

Keywords: Barrier membrane, Stimuli-sensitive, Hydrogel, Controlled release, Nanoparticle

\begin{abstract}
.
A membrane-based approach to pulsatile delivery, termed here as Barrier-Mediated Pulsatile Release (BMPR), sequesters each chemical dose into its own stimuli-sensitive reservoir film, each covered by a stimulant barrier membrane to delay triggering for a prescribed period time. These barrier/depot pairs are then stacked sequentially, with the delay time for the next barrier commencing with the stimulation of the previous depot, assuring a controlled period between each pulse. This paper introduces the first generalized BMPR system, using hydrogel depots that can be adapted to a variety of stimulants, coupled with barriers that rely on sacrificial stimulant scavengers to provide controlled delay times spanning orders of magnitude. Poly(methyl methacrylate-co-dimethylamino ethyl methacrylate) hydrogels are used to demonstrate pulsatile release of multiple solutes triggered by buffered citric acid. Zinc oxide nanoparticles loaded in poly(vinyl alcohol) hydrogel barriers delay acid permeation to each depot. Depot thickness, $\mathrm{pH}$, and buffer strength are each shown to affect the stimulant permeation, swelling, and solute diffusion rates from the depots, with different processes becoming rate-limiting under different conditions. The delay time for each barrier varies linearly with $\mathrm{ZnO}$ scavenger loading, scavenger center-of-mass, and the square of the barrier thickness, providing multiple pathways for tuning delay time over a wide range. BMPR devices releasing up to 10 pulses are demonstrated, with no inherent limit on the number of pulses possible.
\end{abstract}




\section{Introduction}

Many chemicals are most effectively delivered in discrete pulses rather than at a steady rate. Decreased chemical costs and environmental burden are one obvious advantage to only releasing chemicals when actually needed, be they herbicides in fields or biocides on ship hulls. Second, many biological molecules such as hormones are only effective when present in periodic pulses.[1] Vaccines,[2] cancer therapies,[3] and allergen treatments[4] often require repeated pulses in order to most effectively trigger desired biological responses, while other solutes such as nitroglycerin[5] and opiates[6] prompt tolerance within the body, quickly losing effectiveness at constant concentration. Third, many solutes best operate in sequential combinations. For example, one chemical may hinder insects' resistance mechanisms prior to exposure to a second chemical, an insecticide.[7]

Traditional means of achieving pulsatile solute release (manual spreading, spraying, swallowing, or injecting), however, are often impractical. For instance, pills and injections are difficult to administer while asleep. Such administration can be automated with sensors, timers and pumps, though such complexity can quickly become too expensive or unreliable for the application. A delayed pulse can also be achieved by encapsulating an individual dose behind a barrier that degrades or ruptures after a fixed period of time. In theory, one could use an ensemble of such capsules, each with a different degradation time, to achieve periodic pulsatile delivery in a single administration. $[8,9]$ In practice, however, this approach has never been demonstrated beyond three pulses. Since the timing of each capsule is determined independently, small timing errors can soon result in long delays punctuated by overlapping pulses, to say nothing of pulse-smearing within a given capsule set, nor the difficulty in tuning the capsule delay over multiple orders of magnitude. 
This problem can be mitigated, however, by coupling the release of one pulse to the release of the previous one. In this approach, individual doses of solute are interlaced between barriers which delay release only for the additional time to the next pulse. A simple example is depicted in Figure 1, where individual depots of dissolvable chemical are separated by barrier layers of degradable polymer. Upon exposure to water the top polymer barrier erodes, eventually exposing the underlying drug depot which is promptly dissolved and released. At this point water reaches the next polymer barrier layer, eroding it over a similar period of time. It then reaches the next drug depot, releasing a second pulse of drug and triggering the erosion of the third polymer barrier, and so on.
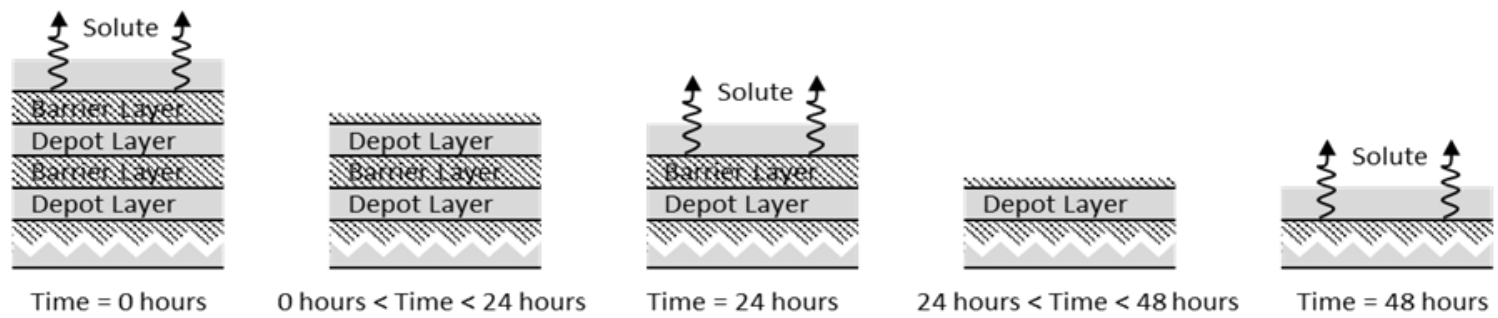

Figure 1: Schematic demonstration of pulsed release from erodible polymer laminate. Upon immersion in water, the first solute pulse is released, followed by a period of no release while the top barrier layer erodes. Once the top barrier layer is gone, a second solute pulse is released, followed by erosion of the next barrier layer.

This approach, which we term Barrier-Mediated Pulsatile Release (BMPR), has been proposed previously,[10-13] but few examples of implementation exist, in part because the above example requires each barrier to be perfectly surface eroding. If any part of the barrier is breached before the rest of it, the breach will release some of the chemical prematurely and broaden the pulse. Also, the delay time of each barrier scales, at best, linearly with the barrier thickness.[14] As the bulk- vs. surface-erosion behavior of polymers also varies with the barrier thickness,[15] time-scaling of the barriers also becomes problematic. Here we report on our efforts to generalize the BMPR approach. By decoupling the delay time of the barriers from 
polymer degradation and triggering the release of solute using stimuli other than water, simple laminates releasing up to ten pre-programmed pulses are demonstrated. Different solutes are released in alternating pulses, and the timing of each pulse can be scaled easily over a wide range.

To generalize the BMPR approach, solid soluble chemical is confined within a stimulisensitive hydrogel. These polymer networks swell with water in response to a stimulant, increasing their permeability dramatically. A wide variety of hydrogel stimulants have been demonstrated, including temperature,[16] $\mathrm{pH},[17]$ glucose,[18] light,[19] electromagnetic fields,[20] and specific antigens.[21] This study employed $\mathrm{pH}$-sensitive methacrylate hydrogels which are glassy at pH 7 but swell with five times their weight in water when subjected to acid at pH 3, as shown in Figure 2. Zinc oxide nanoparticles were used as a sacrificial scavenger within reactive barrier membranes to protect the acid-sensitive depot layers from the acid stimulant for a prescribed period of time. After exhausting the $\mathrm{ZnO}$, the acid triggers the underlying depot layer to swell, delaminating it and its overlying barrier membrane while releasing its payload. The next barrier membrane is then exposed directly to the stimulant and the process repeats.

As the release is decoupled from polymer degradation, the barriers and depots must degrade on a time scale much slower than the chemical release, which may be problematic for some applications. This delamination also adds an additional failure mode which must be addressed for each application. Stimulation by $\mathrm{pH}$, however, opens this approach to many other potential stimulants such as hydrocarbons, which can be converted to organic acids in situ. Bacterial biofilms in oil tanks, for instance, generate acids which are harmful to downstream equipment. Individual biocide pulses could automatically mitigate these biofilms when their acid generation becomes problematic. 


\section{Materials \& Methods}

\subsection{Materials}

Methyl methacrylate (MMA), 2dimethylamino ethyl methacrylate (DMA), and divinyl benzene (DVB) were obtained from Aldrich (Milwaukee, WI). Polymerization inhibitors in these monomers were removed by adsorption on



Figure 2: Poly(methyl methacrylate-co-dimethylamino ethyl methacrylate) disk at $\mathrm{pH}=7$ (left) and $\mathrm{pH}=3$ (right). Under neutral conditions the polymer is glassy and hydrophobic, effective encapsulating water-soluble drug powders. Under acidic conditions the polymer swells with water, dissolving and releasing drug. Aqueous blue dye was added for contrast.

alumina (Fisher Scientific, Pittsburgh, PA), the monomers were then refrigerated until used. 2, 2'-Azobisisobutyronitrile (AIBN), zinc oxide ( $\mathrm{ZnO})$, citric acid, sodium hydroxide, and potassium chloride were obtained from Sigma-Aldrich and used as received. Monobasic potassium phosphate and sodium chloride were used as received from Research Products International (Mt. Prospects, IL). Poly (vinyl alcohol) (PVA, 99\% hydrolyzed, MW 133,000) was obtained from Polysciences (Warrington, PA) and used as received. Methylene blue (MB, Sigma-Aldrich) and methyl orange (MO, Alfa-Aesar, Wardhill, MA) were ground with a mortar and pestle then screened at 100 mesh before use.

\subsection{Device fabrication}

\section{$\underline{\text { 2.2.1 Depot layer fabrication }}$}

Poly (methyl methacrylate) (PMMA) is a well-studied polymer with excellent mechanical properties and a wide range of commercial applications. Incorporation of acid-sensitive comonomers into PMMA has been previously studied, with dimethylamino ethyl methacrylate (DMA) in a 70/30 (by mol) MMA/DMA ratio retaining both the glassy, hydrophobic character of PMMA at neutral $\mathrm{pH}$ and showing a strongly hydrophilic swelling behavior at $\mathrm{pH} 6$ and 
below.[17, 22, 23] This impermeable "off-state" in the absence of stimulus is a critical feature for BMPR depot materials, as it allows large doses of solid soluble chemical to be loaded into each depot and retained for long periods of time without significant degradation or leakage. It also complicates control of the depot swelling rate, however, as discussed in Section 3. By incorporating a co-monomer or cross-linking agent which is hydrolytically or enzymatically cleaved, biodegradable pH-sensitive PMMA gels have also been produced.[24]

To create chemical-loaded poly (methyl methacrylate-co-dimethylamino ethyl methacrylate) (PMMA/DMA) films, $3.909 \mathrm{~mL}$ of DMA was added to $6.315 \mathrm{~mL}$ MMA, $10.5 \mu \mathrm{L}$ divinyl benzene crosslinker, and $48.03 \mathrm{mg}$ 2,2'-azobisisobutryonitrile (AIBN) in a $20 \mathrm{~mL}$ vial. After heating to $70{ }^{\circ} \mathrm{C}$ with stirring, the solution polymerized to a viscosity of approximately 65 cP before being chilled to room temperature. Methylene blue (MB) powder (a model drug released in these studies) was then dispersed into the oligomer solution by stirring and sonication (Cole-Parmer CPX750 with $3.2 \mathrm{~mm}$ microtip, $1 \mathrm{~min}$ at $300 \mathrm{~W}, 20 \mathrm{kHz}$ ). The suspension was then poured between two silanized glass plates, with poly (tetrafluorethylene) (PTFE) spacers clamped between them. Different spacers were used to make films of different thicknesses from 50 to $350 \mathrm{um}$. The plates were then mounted vertically in a vacuum oven at $60{ }^{\circ} \mathrm{C}$ under nitrogen for 18 hours, after which the MB-loaded polymer film was cooled, removed from the glass plates, and stored under ambient conditions until used. Thickness variation across an 18$\mathrm{cm}$ wide film could exceed $20 \mu \mathrm{m}$ due to the negative volume of polymerization in this closed system, though individual depots cut with a 15-mm-wide cork borer had thickness variation of less than $2 \mu \mathrm{m}$.

\subsubsection{Barrier layer fabrication}


Reactive acid barrier membranes using zinc oxide dispersions in poly (vinyl alcohol) (PVA) have been described previously.[25, 26] Briefly, 15 g PVA were dissolved in $135 \mathrm{~mL}$ water at $90{ }^{\circ} \mathrm{C}$ under stirring. Zinc oxide nanoparticles corresponding to the desired dry weight percentage (10-40\%) were added and dispersed through stirring and sonication. The large surface area of the $\mathrm{ZnO}$ nanoparticles assured rapid scavenging of any passing stimulant acid, as well as homogenous distribution of the $\mathrm{ZnO}$ even at very thin film thicknesses. The suspension was then cooled to room temperature and cast on a PTFE sheet, followed by calendaring with a custom-built doctor blade to the desired thickness. After drying for at least 24 hours under ambient conditions, the films were peeled off, clamped between two glass plates, and cured at $150{ }^{\circ} \mathrm{C}$ under vacuum for two hours to crosslink. Films were stored under ambient conditions then immersed in water to hydrate at least 24 hours before use. After hydration, individual barriers with a thickness variation of less than $3 \mu \mathrm{m}$ were cut using a 16-mm-wide cork borer. Barrier-to-barrier thickness variation from a given 10-cm-wide coating was larger, however, due to a variety of issues associated with calendaring viscous suspensions.

\section{$\underline{\text { 2.2.3 Device fabrication }}$}

Disks of dry PMMA/DMA depot film $15 \mathrm{~mm}$ in diameter were stacked alternately with $16 \mathrm{~mm}$ diameter disks of hydrated PVA barrier film atop a $0.5 \mathrm{~mm}$ thick, $16 \mathrm{~mm}$ diameter PTFE plug. The plug was placed within a circular PTFE mold $6.7 \mathrm{~mm}$ thick and $135{ }^{\circ} \mathrm{C}$ beeswax was poured into the mold, surrounding and covering the polymer stack. After freezing for $15 \mathrm{~min}$, the PTFE plug was removed, leaving a simple coin-shaped laminate pulsatile release device.

The primary challenge in device fabrication is reliably achieving complete contact between the wax and every hydrated barrier layer, to avoid stimulant leakage around the edge of the barrier. Besides making the barrier layers slightly wider than the depots and recessing the 
stack slightly into the wax sidewall using the PTFE plug, a hydrophobic PMMA/DMA layer was placed on the outer face of every device, even when an immediate solute pulse was not desired. In those instances, this outer depot layer contained no payload and effectively acted as simply another barrier layer.

\subsection{Device characterization}

\subsubsection{Depot layer characterization}

To confirm the dimethyl amino ethyl methacrylate (DMA) content of the depot membranes, dry samples (40 mg average weight) were individually equilibrated in $150 \mathrm{mM}$ saline solutions ( 300 times sample weight) initially set to $\mathrm{pH} 2$ via $\mathrm{HCl}$ addition. Upon addition of the polymer sample, the $\mathrm{pH}$ of each solution rose to $2.76\left({ }^{ \pm} 0.06\right.$ on $\left.\mathrm{n}=3\right)$. At equilibrium, all amine groups in this polymer are protonated in this $\mathrm{pH}$ range,[27] allowing the MMA:DMA mol ratio in the depot membranes to be stoichiometrically calculated as 75:25.

The swelling rate and equilibrium swelling ratio of drug-free PMMA/DMA membranes were characterized against three parameters: $\mathrm{pH}$, membrane thickness, and buffer concentration (neutral weak acid + conjugate base). Circular disks (12 mm diameter) of PMMA/DMA membrane were immersed in $250 \mathrm{~mL}$ buffer and periodically reweighed.

For pH-dependency trials, $223 \mu \mathrm{m}\left({ }^{ \pm} 1.7 \%\right.$ RSD) thick membranes were immersed in

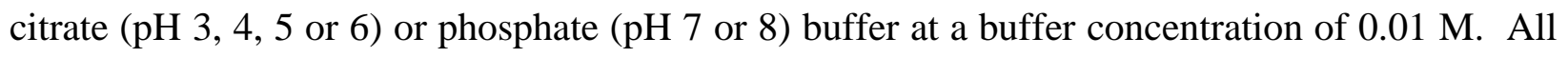
buffers used a sodium counter-ion and were set to an ionic strength of $0.1 \mathrm{M}$ by $\mathrm{NaCl}$ addition unless otherwise indicated. To investigate the effect of membrane thickness on swelling rate and equilibrium swelling ratio, membranes ranging in thickness from 110 to $350 \mu \mathrm{m}$ were tested at $\mathrm{pH}$ 3. These trials were performed at buffer concentrations of both $0.01 \mathrm{M}$ and $0.1 \mathrm{M}$ to demonstrate the effect of buffer concentration. All trials were performed in triplicate. 
Additional swelling information was gained in conjunction with release rate trials using PMMA/DMA disks loaded with 5\% (w/w) methylene blue powder. For these trials, depots ranging in thickness from 75 to $350 \mu \mathrm{m}$ were tested first in $\mathrm{pH} 7$ buffer, then transferred to 0.01 M pH 3 buffer. Swelling was measured gravimetrically as above, while release of methylene blue was measured by a UV/Vis spectrophotometer (Varian Cary 50, Mulgrave, Australia) equipped with an immersion probe.

The transport properties of both the depot and barrier membranes were measured using a diaphragm cell. Briefly, the film was sandwiched between two well-stirred $22 \mathrm{~mL}$ cups. The upstream cup was spiked with solute and the downstream cup's solute concentration was measured vs. time to determine a steady-state flux, from which the membrane's permeability was calculated. For methylene blue permeability trials, depot films were soaked in $0.01 \mathrm{M}$ buffer at $\mathrm{pH} 3$ or $\mathrm{pH} 7$ for 1-3 days prior to testing. Additional trials doubling the stirring speed in the diaphragm cell reservoirs confirmed negligible mass transfer resistance between the membrane and the adjacent reservoir liquids.

Acid transport across the depot membrane is inherently more difficult to characterize, in part because the membrane's transport properties are $\mathrm{pH}$-dependent. For these studies, a titrator (Metrohm Titrando 842, Herisau, Switzerland) maintained the downstream $\mathrm{pH}$ at 4.0 (initial citrate buffer strength $0.001 \mathrm{M}$ ) while recording the rate of sodium hydroxide addition required to maintain this $\mathrm{pH}$ across from an upstream citrate buffer (initial buffer strength $0.01 \mathrm{M}$ ) at $\mathrm{pH}$ 3. The rate of $\mathrm{NaOH}$ addition to maintain constant downstream $\mathrm{pH}$ indicated the rate of total acid transport across the membrane. Prior to testing, the membranes were soaked in $\mathrm{pH} 4$ citrate buffer (buffer strength $0.001 \mathrm{M}$ ) for three days, making their initial acid concentration comparable to the downstream reservoir. 


\subsubsection{Barrier Layer Characterization}

To confirm that the $\mathrm{ZnO}$ content of the barrier membranes matched their recipe, $20 \mathrm{mg}$ (dry weight) samples were individually placed in $100 \mathrm{~mL}$ of $0.001 \mathrm{M} \mathrm{HCl}$. As the $\mathrm{ZnO}$ in the membrane consumed the acid, additional $0.001 \mathrm{M} \mathrm{HCl}$ was metered into each container to maintain a target $\mathrm{pH}$ of 3.5 using an automatic titrator. The difference between the final hydronium ion content and the initial and added hydronium content indicated the amount of $\mathrm{ZnO}$ in the sample, with each $\mathrm{ZnO}$ unit consuming two hydronium ions. The average discrepancy in $\mathrm{ZnO}$ weight fraction between the fabrication recipe and subsequent titration was $0.3 \%$ across three separate membranes.

This demonstrated that the $\mathrm{ZnO}$ concentration of each dry barrier membrane can be precisely controlled by its fabrication recipe, however it is the $\mathrm{ZnO}$ concentration in the barrier membrane's swollen state that determines its performance. To characterize barrier membrane swelling, dry barrier membranes of various $\mathrm{ZnO}$ weight fraction $(0-80 \%)$ and thickness $(39$ to $167 \mu \mathrm{m})$ were swollen in deionized water for several days, then reweighed.

To confirm that the $\mathrm{ZnO}$ did not settle to one face of the barrier membrane during fabrication, membrane cross-sections were inspected by secondary electron microscopy. Figure 3 shows SEM images of a PVA film with no $\mathrm{ZnO}$ (A), a PVA film containing $10 \%$ (dry weight basis) $\mathrm{ZnO}(\mathrm{B})$, and a PVA film in which the $\mathrm{ZnO}$ is distributed through only half the PVA thickness (C). The latter film was obtained by casting a $\mathrm{ZnO}$-free PVA layer atop a previously cast and dried ZnO-laden PVA layer, prior to cross-linking. While the SEM images indicate qualitatively the homogenous dispersion of the $\mathrm{ZnO}$, slight asymmetry in the $\mathrm{ZnO}$ distribution (i.e., settling) is more difficult to detect with this method. Such asymmetry will, however, 
noticeably alter the lag time of acid across the film, with faster acid breakthrough occurring if the scavenger has settled toward the upstream face of the membrane.[26]
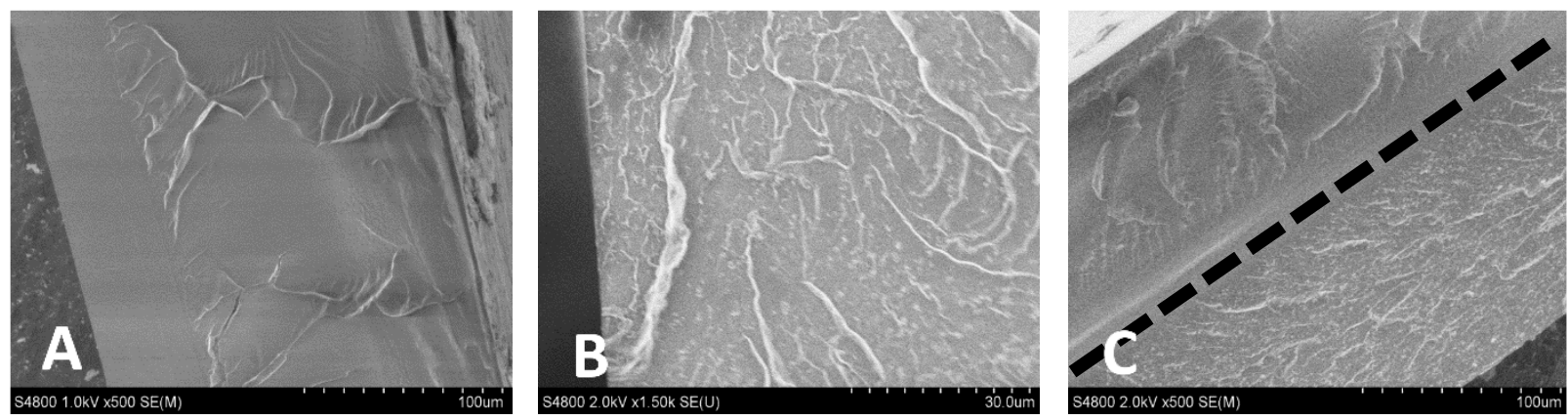

Figure 3: SEM cross-sectional images of PVA barrier films. (A) without $\mathrm{ZnO}$ scavenger, (B) with $10 \%$ (w/w) $\mathrm{ZnO}$ scavenger, (C) as a bilayer with no $\mathrm{ZnO}$ in the upper-left and $10 \%(\mathrm{w} / \mathrm{w}) \mathrm{ZnO}$ in the lower right (dashed line added at bilayer interface).

To test for such asymmetry, as well as measure the transport properties of the barrier membranes, diaphragm cells studies as described for the depot membranes were also performed on the barrier membranes. These experiments used an upstream $0.01 \mathrm{M} \mathrm{pH} 3$ citrate buffer with a downstream solution of $100 \mathrm{mM} \mathrm{NaCl}$ at neutral $\mathrm{pH}$. Membranes with initial $\mathrm{ZnO}$ weight fractions (dry basis) ranging from 0 to $60 \%$ were tested, with replicates oriented in opposite directions to detect any settling of the $\mathrm{ZnO}$. The barriers ranged in thickness from 72 to $520 \mu \mathrm{m}$, with the thickest barriers comprised of multiple layers of thinner membranes in series. Select $\mathrm{ZnO}$-laden films were soaked in deionized water for at least three days after testing, then retested to observed their acid lag-time and permeability after removal of the $\mathrm{ZnO}$. Due to the high acid scavenger content of some barriers, the upstream reservoir was titrated as needed with $0.1 \mathrm{M}$ $\mathrm{HCl}$ to maintain its $\mathrm{pH}$ at 3 . Further diaphragm cell studies determined the permeability of methylene blue and methyl orange across $\mathrm{ZnO}$-free PVA films ranging in thickness from 92 to $373 \mu \mathrm{m}$. These trials used $0.01 \mathrm{M} \mathrm{pH} 3$ citrate buffer on both sides, with the target solute spiked upstream.

\subsection{Device testing}


The release behavior of each device was determined by taping it to a glass platform and immersing it in $>0.3 \mathrm{~L}$ of $0.01 \mathrm{M}$ buffer. Phosphate $(\mathrm{pH} 7)$ and citrate $(\mathrm{pH} 3)$ buffers were used for these trials, with the $\mathrm{pH}$ monitored by $\mathrm{pH}$ meter and the UV/Vis spectrum of the buffer solution periodically scanned using an immersed fiber-optic probe coupled to a UV/Vis spectrophotometer. Pictures of the buffer and immersed device were recorded every 15 minutes by a PC-interfaced camera. Buffer solution was added and/or replaced as needed to keep the optical absorbance of relevant wavelengths below 1.0, also ensuring that the buffer composition did not drift significantly.

\section{Results and Discussion}

\subsection{Depot layers}

One critical feature of BMPR depots is an "off state" in which the chemical release rate is negligibly small. This criterion immediately eliminates some of the most popular stimuli-sensitive polymers, most notably acrylamide copolymers, which even in their shrunken

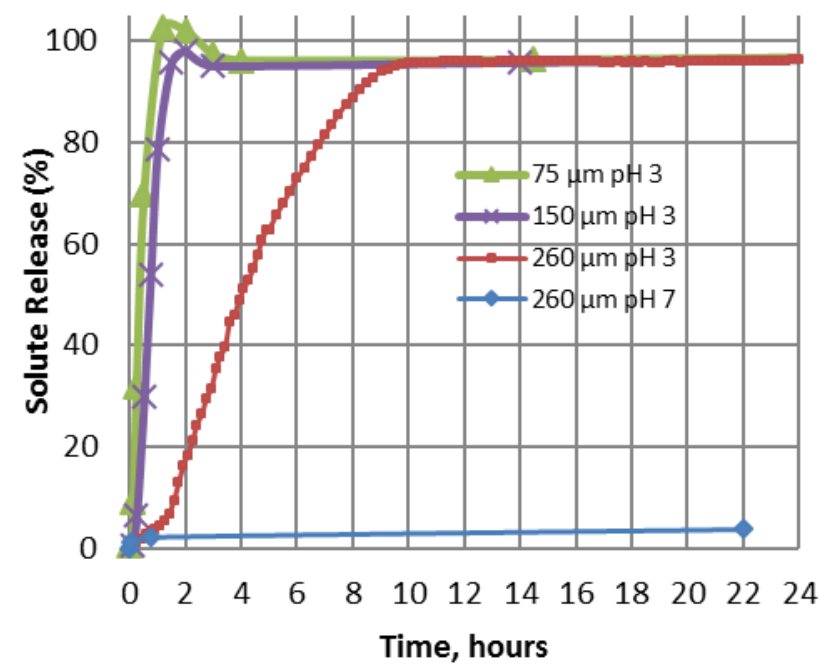

Figure 4: Solute Release from PMMA/DMA hydrogels. No release at $\mathrm{pH} 7$ except for small $(\sim 4 \%)$ initial burst due to film cutting. Prompt, complete release at $\mathrm{pH} 3$.

hydrophobic state are rubbery and contain a non-negligible amount of water. Figure 4 demonstrates that PMMA/DMA hydrogels meet this criteria. Their extreme hydrophobicity and glassy structure at neutral $\mathrm{pH}$ effectively block the dissolution and diffusion of all encapsulated drug; only $\sim 4 \%$ of the solute was released at $\mathrm{pH} 7$, and that drug was released instantly, likely because it was directly exposed to the buffer solution around the perimeter of the disk where it was cut. A second critical feature is a highly permeable "on state" in which the chemical can 
rapidly dissolve and diffuse away. This is also shown in Figure 4, where at $\mathrm{pH} 3$ nearly all of the drug in the thinner depots is released in the first hour of exposure, despite a day without release at $\mathrm{pH} 7$.

The depot swelling trials indicate that the swelling is strongly $\mathrm{pH}$-dependent and, in most cases, transport-limited. This is in good agreement with previous studies of PMMA/DMA hydrogels.[22, 28] The sigmoidal shape of the swelling curves in Figure 5 (shown more clearly in the inset)

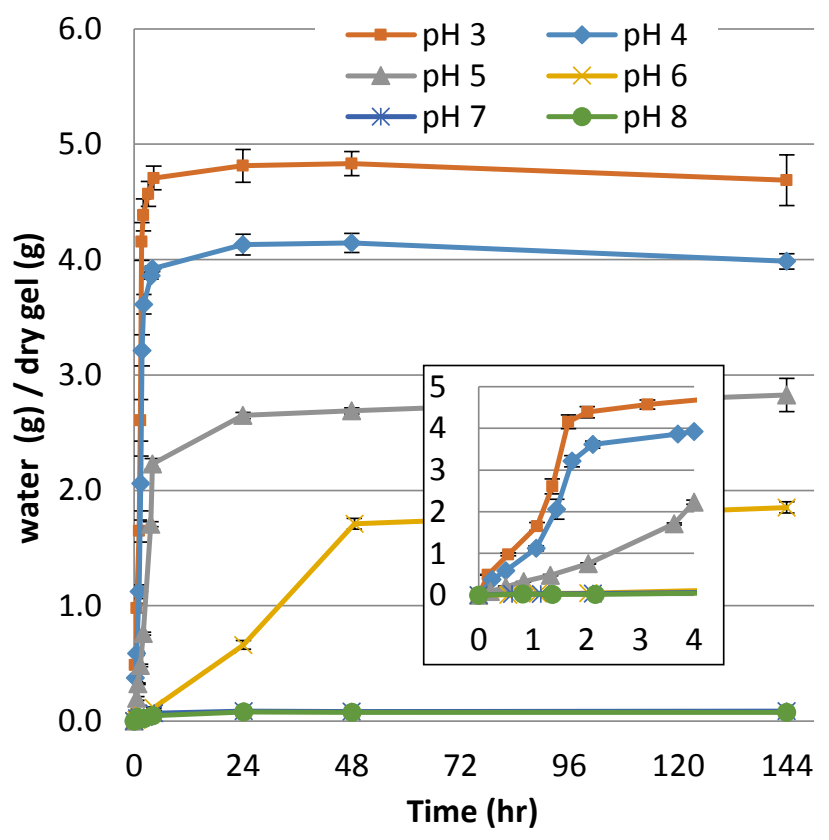

Figure 5: Swelling of PMMA/DMA hydrogels. Equilibrium swelling decreases with increasing $\mathrm{pH}$ and appears negligible at $\mathrm{pH} 7$. Error bars show standard deviation with $n=3$. Sigmoidal shape of swelling curve at short times (inset) shows that swelling rate is transport-limited. indicate a swelling front advancing from each face of the membrane towards the center. As the fronts move deeper into the membrane, the rate of acid diffusing to the front to advance it decreases, slowing its progress and hence the swelling rate. When the fronts meet at the center of the membrane, however, swelling is no longer confined to one dimension and the membrane rapidly swells in the other two dimensions before reaching equilibrium. Figure 6 further demonstrates that the swelling is $\mathrm{pH}$-dependent and transport limited by comparing swelling rates at pH 3 and 7 for depots of different thickness. None of the membranes swell at all at pH 7 and all membranes swell rapidly at $\mathrm{pH}$ 3. Moreover, all but the thinnest membranes show sigmoidal swelling curves as well as swelling rates that vary with the square of the membrane thickness. Notably, the thinnest membranes (112 $\mu \mathrm{m}$ average) do not have a discernable sigmoidal shape to their swelling curve, and their overall swelling time is not four times shorter 
than the $230 \mu \mathrm{m}$ membranes', despite being twice as thin. The swelling still takes roughly 30 minutes, suggesting that this is a polymer relaxation limit, consistent with earlier studies.[28]

While Figures 5 and 6 demonstrate that the swelling rate is $\mathrm{pH}$-dependent and diffusion-limited, Figure 7 indicates that this is not due to larger $\mathrm{H}^{+}$concentrations

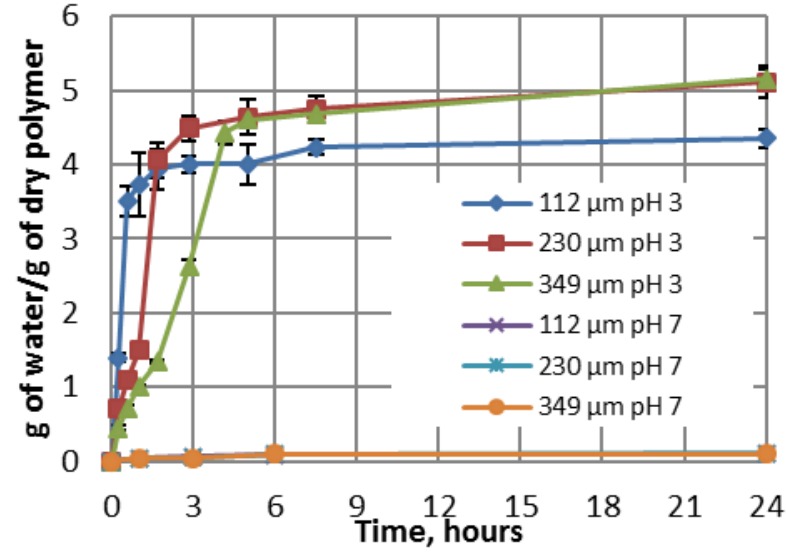

Figure 6: Thickness dependence of swelling P(MMA/DMA) hydrogels. All three $\mathrm{pH} 7$ lines overlay each other with no significant swelling. At $\mathrm{pH} 3$ swelling rate varies with film thickness. Note inflection in swelling curves of films more than $150 \mu \mathrm{m}$ thick.

driving faster $\mathrm{H}^{+}$diffusion. Rather, at uniform buffer strength the concentration of neutral weak acid increases strongly with decreasing $\mathrm{pH}$, and it is diffusion of this neutral weak acid that controls the swelling rate. When the buffer strength is increased from $0.01 \mathrm{M}$ to $0.1 \mathrm{M}$ at the same $\mathrm{pH}$ (3), the swelling rate increases dramatically. This effect has been explored

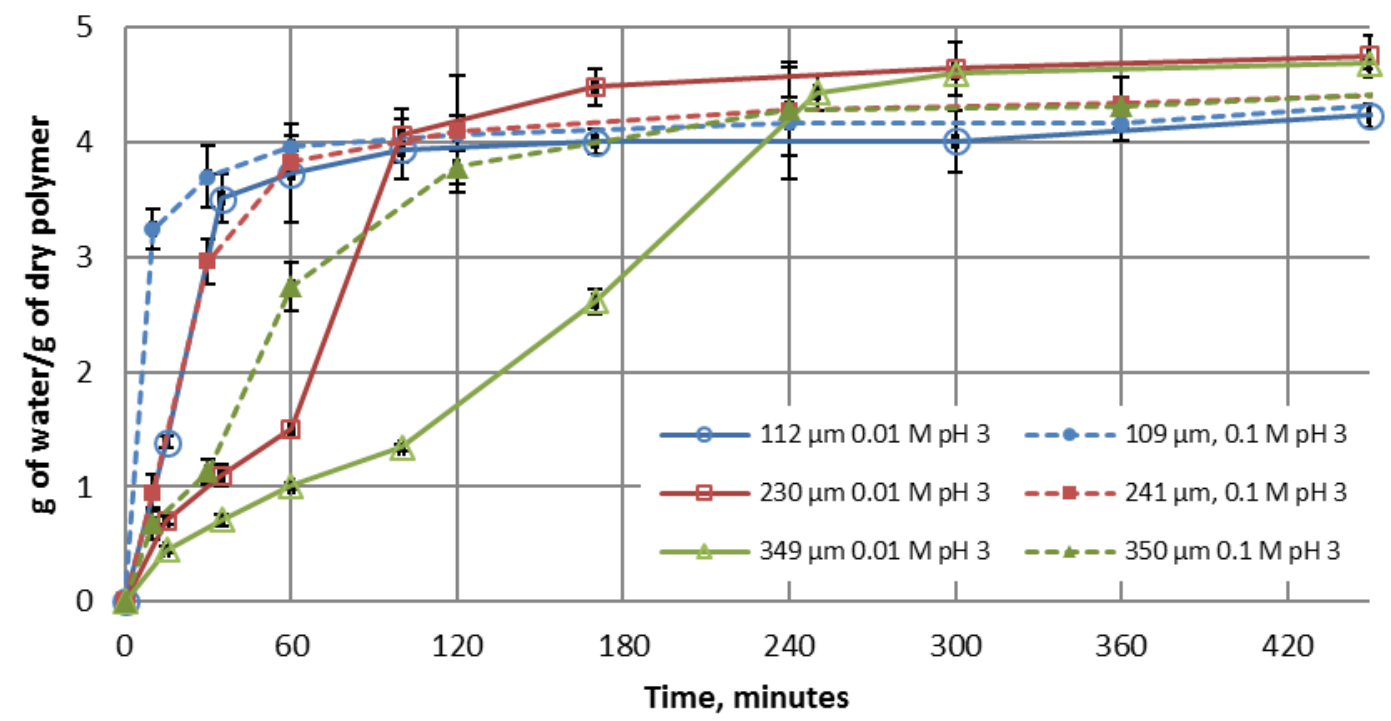

Figure 7: Effect of buffer strength and film thickness on swelling PMMA/DMA hydrogels. At any thickness, films swell significantly faster in more concentrated buffer at the same $\mathrm{pH}$, underscoring the importance of the neutral weak acid in these systems. 
previously[27] and can be explained by the strong hydrophobicity of the shrunken gel. While $\mathrm{H}^{+}$ can diffuse very rapidly in aqueous media, it cannot penetrate into the hydrophobic region of the polymer and is therefore limited to protonating the amines right at the interface of the hydrophobic region, advancing that interface very slowly. By contrast, a neutral organic acid can partition into the hydrophobic region, then dissociate when it encounters an amine. Being able to protonate amines beyond the hydrophobic interface, it can advance that interface much more rapidly. This was most starkly demonstrated by swelling trials in $\mathrm{HCl}$ at $\mathrm{pH} 3$, where the swelling time was measured in days rather than minutes. The $0.1 \mathrm{M}$ buffer trials

in Figure 7 also further demonstrate the second-order dependence of swelling on membrane thickness. Moreover, even the thinnest membranes swell faster at a higher buffer strength, indicating that diffusion is still significant, though not completely rate-limiting, under those conditions.

This has significant implications for adapting BMPR systems with $\mathrm{pH}$-sensitive hydrogels to specific applications. For instance, while the digestive tract maintains a strongly acidic environment, gastric acid is unlikely to be an effective stimulant for this sort of hydrogel (assuming all other barriers to digestive tract implementation were overcome). Enzymatic conversion of blood glucose to gluconic acid, on the other hand, may provide a viable stimulant, despite external $\mathrm{pH}$ buffering by the blood. The presence of a particular external $\mathrm{pH}$ is relevant only if it influences the flux of neutral acid species that can be delivered, an influence that varies with the $\mathrm{pK}_{\mathrm{a}}$ of the acid and the location at which the acid is generated.

The neutral acid flux also plays a prominent role in the diaphragm cell transport studies. As discussed in the Supplemental Information, its diffusion coefficient is generally smaller than that of dissociated protons, but its concentration gradient from $0.01 \mathrm{M} \mathrm{pH} 3$ citrate buffer is 
larger, resulting in comparable fluxes of dissociated and neutral acid. By titration only their combined flux is measured, at $1.1 \times 10^{-9} \mathrm{~mol} /\left(\mathrm{cm}^{2} \mathrm{~s}\right)$ for $500 \mu \mathrm{m}$ of membrane, scaling inversely with membrane thickness. Since the two species have different concentration gradients, a meaningful permeability cannot be directly calculated. By comparison, however, the combined flux across a hypothetical $500 \mu \mathrm{m}$ membrane of pure water between those reservoirs would be $1.3 \times 10^{-9} \mathrm{~mol} /\left(\mathrm{cm}^{2} \mathrm{~s}\right)$, only $20 \%$ higher. That hypothetical pure water membrane would also have $20 \%$ more water than the hydrogel membrane, as the polymer occupies $15-20 \%$ of the membrane volume at equilibrium in those conditions, as indicated in Figure 5. In short, the acid species appear to diffuse across the swollen depot membrane with the same diffusion coefficients as in water and with partition coefficients equal to the hydrogel's water volume fraction.

While the transport coefficients for small stimulant molecules through the swollen depot may be estimated from their coefficients in water, the diaphragm cells studies on methylene blue and methyl orange yield a different result. Here the depot films were sandwiched between 0.01 $\mathrm{M} \mathrm{pH} 3$ buffer on both sides, with a spike of dissolved drug added to the upstream cell. The permeabilities of the methylene blue and methyl orange through PMMA/DMA were $3.4 \times 10^{-7}$ $\mathrm{cm}^{2} / \mathrm{s}$ and $3.0 \times 10^{-7} \mathrm{~cm}^{2} / \mathrm{s}$, respectively. Using the lag times of these trials, the diffusion coefficient of methylene blue in the depot film was calculated at $7.1 \times 10^{-7} \mathrm{~cm}^{2} / \mathrm{s}$, yielding a partition coefficient of 0.43 . This was confirmed by equilibrating depot films in a $\mathrm{pH} 3$ buffer of known methylene blue concentration, then desorbing the solute into solute-free buffer for quantification, yielding a partition coefficient of 0.48. Unlike acid, methylene blue avoids entering the depot (partition coefficient less than the gel's water volume fraction) and diffuses more slowly in the gel than it does in water $\left(\sim 7 \times 10^{-6} \mathrm{~cm}^{2} / \mathrm{s}\right)$,[29] perhaps owing to its larger size (MW $320 \mathrm{~g} / \mathrm{mol}$ ), planar configuration, and positive charge. Since the depot will serve as an ion 
exchange resin for the negatively charged methyl orange, the distinction between partitioning and adsorption becomes blurred. Its low permeability, however, suggests that it also diffuses more slowly in the gel than in water. Its size (MW $327 \mathrm{~g} / \mathrm{mol}$ ) and configuration (planar) are comparable to methylene blue's. No measurable solute transport was observed in diaphragm cell studies at pH 7 for either solute.

Despite these slower mass-transport coefficients, the release rate of chemical leaving the depot appears to be on par with the swelling rate, as shown in Figure 8 which plots both swelling and chemical release vs. time for depots of different thicknesses. Besides noting that the equilibrium swelling ratio for methylene blue loaded depots is higher than for the drug-free depots of Figure 5 (methylene blue inhibits polymerization,[30] likely prompting a looser network), Figure 8 also shows that the time required for the depot to release its payload is approximately twice its swelling time regardless of the film thickness, indicating that solute release is also transport limited.

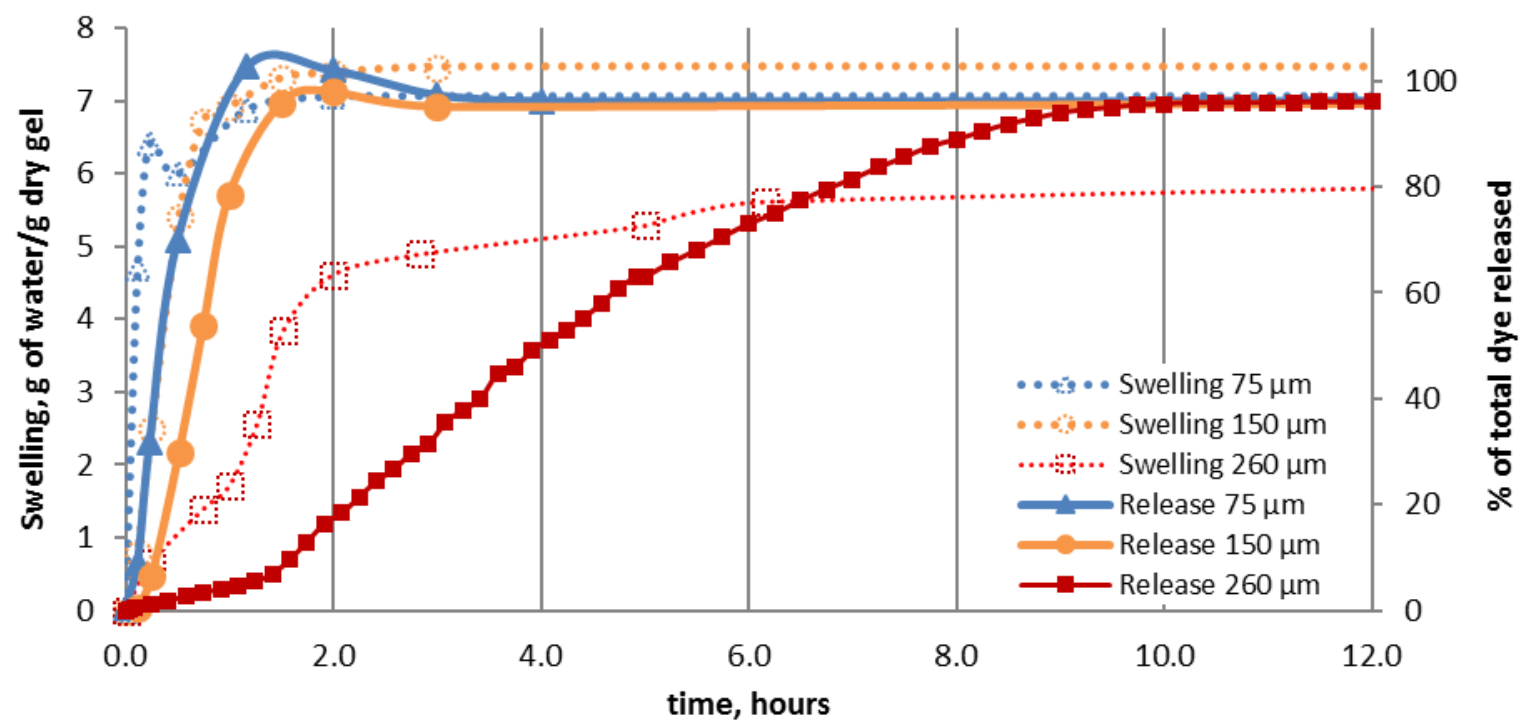

Figure 8: Comparison of swelling and solute release rates in PMMA/DMA hydrogels. No swelling and negligible release of methylene blue at $\mathrm{pH} 7$. At $\mathrm{pH} 3$ the characteristic swelling and release times can be estimated analytically. 
This suggests that one can adapt Higuchi's transport equations[31, 32] to estimate characteristic times for both swelling $\left(\mathrm{t}_{\mathrm{swell}}\right)$ and chemical release $\left(\mathrm{t}_{\mathrm{rel}}\right)$ :

$$
t_{\text {swell }}=\frac{c_{\text {seav }}}{c_{\text {stim }}} \frac{l^{2}}{2 D H_{\text {stim }}} \quad t_{\text {rel }}=\frac{C_{\text {solid }}}{C_{\text {sat }}} \frac{l^{2}}{2 D H_{\text {solute }}}
$$

$C_{\text {stim }}$ and $C_{\text {sat }}$ are the external acid concentration and the drug saturation concentration, respectively, while $C_{\text {scav }}$ and $C_{\text {solid }}$ are the concentration of acid-scavenging amine groups and solid chemical in the depot, respectively. $D H$ is the permeability of the indicated species in the depot, and $\ell$ is the overall distance across which transport must occur. For a depot with homogeneously dispersed chemical, release starts at the same time as swelling, but clearly cannot finish before the swelling is complete, so $t_{\text {rel }}<t_{\text {swell }}$ simply means that the release is limited by the depot's swelling rate. For $t_{\text {rel }}>t_{\text {swell }}$, however, release will continue significantly after the depot has swollen and delaminated, while stimulant is penetrating the next barrier membrane. If $t_{r e l}-t_{\text {swell }}$ is longer than the lag time of the next barrier, the next pulse may begin before the previous pulse has finished, resulting in continuous release if the chemicals in both depots are the same.

For the depot membranes in Figure $8, \ell$ is the half-thickness of the membrane in the swollen state. While this transport is occurring the swelling is constrained to one dimension by the not-yet-swollen center of the film. From the sigmoidal curves in Figures 5-8 we see that the membranes swell by a factor of two before the one-dimensional constraint is lifted and the membrane swells more rapidly. This is verified by PMMA/DMA membranes covalently bound to glass substrates to permanently restrict swelling to one dimension. At equilibrium in the same pH 3 citrate buffer they double in both size and thickness. Doubling the half-thickness of each membrane in Figure 8, $\ell$ ends up being equal to the dry thicknesses listed in the figure legend. The amine concentration and solid drug concentration for those membranes under those 
conditions are $1.17 \mathrm{M}$ and $0.073 \mathrm{M}$, respectively, and the methylene blue saturation concentration determined in Section 2 is $5.9 \mathrm{mM}$. Using the permeabilities and acid concentrations discussed earlier, $\mathrm{t}_{\mathrm{swell}}$ for depot membranes with dry thicknesses of 75,150 , and $260 \mu \mathrm{m}$ are $0.17,0.66$, and 1.99 hours, respectively, matching the curves in Figure 8 quite well. The characteristic release times for these membranes are comparable: $0.28,1.14$, and $3.41 \mathrm{hrs,}$ respectively, indicating that both rate processes influence the final release profile in these release trials. To ensure that the release from sequential depots is pulsatile, the lag time of the intervening barrier membrane must be comparable.

\subsection{Barrier Layers}

Ideally, the barrier layer blocks stimulant from reaching the depot layer for a fixed period of time, then allows the stimulant into the depot unhindered. To achieve this ideal, the barrier must have a large innate permeance to the stimulant while simultaneously having a large stimulant-scavenging capacity. The characteristic lag time $\left(t_{L}\right)$ for stimulant permeation across a barrier loaded with a sacrificial scavenger is $[26,31,33]$

$$
t_{L}=\frac{I^{2}}{6 D}\left(1+\frac{6 \mathrm{C}_{\mathrm{scav}} z_{m}}{\mathrm{v} H C_{\mathrm{stim}}}\right)
$$

where $C_{\text {scav }}$ is now the concentration of acid-scavenging $\mathrm{ZnO}$ groups (rather than amine groups, as in the depot layer) and $v$ is a stoichiometric coefficient (in this case, $1 / 2$ since $1 / 2$ of a $\mathrm{ZnO}$ scavenger consumes a proton). $Z_{m}$ is the location of the scavenger's center of mass across the film; for homogenously distributed scavenger, the center of mass is midway across the film and $Z_{m}=0.5$. Equation 3 is essentially a more precise version of Equation 1 which accounts for heterogeneity in scavenger distribution and the tiny amount of stimulant still needed when $C_{\text {scav }}$ approaches zero. Like Equation 1 it shows that the lag time varies with both the concentration of scavenger and with the square of the film thickness. These relationships are demonstrated in 
Figure 9, where breakthrough curves of integrated acid flux vs. time are plotted for PVA films with $\mathrm{ZnO}$ content ranging from 0 to $30 \%$ (dry weight basis) and thicknesses ranging from 72 to $224 \mu \mathrm{m}$. The lag time for $\mathrm{ZnO}$-free films is on the order of a minute, while a $94-\mu \mathrm{m}$-thick film with $10 \% \mathrm{ZnO}$ by weight has a lag time of $1.64 \mathrm{hrs}$. Based on Equation 3, the lag times for the $20 \%$ and $30 \% \mathrm{ZnO}$ films should be larger by the ratio of $\mathrm{ZnO}$ fractions times the square of the ratio of film thicknesses, to 1.92 and 3.99 hrs, respectively. Experimentally, these films had lag times of 1.86 and $3.97 \mathrm{hrs}$, respectively. Stacking three $20 \% \mathrm{ZnO}$ films together, the lag time increases to $15.63 \mathrm{hrs}$, within $7 \%$ of the expected factor of $3^{2}$ increase.



Figure 9: Breakthrough curves for acid across barrier films. Lag time prior to acid breakthrough increases linearly with $\mathrm{ZnO}$ concentration and with the square of film thickness. Acid permeance, as indicated by the breakthrough slope, is independent of $\mathrm{ZnO}$ loading and varies only inversely with barrier thickness.

While the lag time increases by an order of magnitude, however, the permeability remains unchanged. Diaphragm cell trials indicate an acid permeability across the barrier membranes of $1.47\left({ }^{ \pm} 0.1\right) \times 10^{-5} \mathrm{~cm}^{2} / \mathrm{s}$. As PVA has little $\mathrm{pH}$ sensitivity, acid flux in these trials was measured directly from $\mathrm{pH}$ shift in the unbuffered downstream reservoir, spanning into a $\mathrm{pH}$ range where the flux of neutral acid species contributes little $(\sim 12 \%)$ to the measured shift. This 
was confirmed by trials using unbuffered citric acid to operate in a lower $\mathrm{pH}$ range where even less of the neutral acid flux would dissociate downstream; under those conditions the observed permeability was only $9 \%$ lower. The importance of this result is to demonstrate that despite being able to completely block the stimulant for a long, prescribed period of time, the barrier membrane still has a very large stimulant permeability, comparable to pure water. Swelling studies demonstrated that the membrane (excluding the scavenger) is $55 \%$ water by volume. The acid permeability listed above is $46 \%$ of the acid permeability through pure water. This suggests that, like the depot membrane, acid permeates the barrier membrane nearly as it would through pure water, unaffected by the surrounding polymer superstructure. Moreover, initial incorporation of $\mathrm{ZnO}$ should have little effect on permeability, both because the volume of the $\mathrm{ZnO}$ is so small ( $<10 \%$ by volume for a membrane initially containing $50 \%$ (dry weight basis) $\mathrm{ZnO}$ ) and because once the $\mathrm{ZnO}$ is consumed that volume is filled by a medium (water) with a similar permeability. Also like the depot membranes, the barrier membranes' permeability for larger molecules such as methylene blue is affected by the polymer network. Diaphragm cell studies indicate a methylene blue permeability across the barrier membranes of $3.57 \times 10^{-7} \mathrm{~cm}^{2} / \mathrm{s}$, only $10 \%$ of its permeability across a comparable amount of pure water.

\subsection{BMPR Devices}

Relying on depot swelling as the triggered action for solute release, it is critical that the depots be completely shrunken and hydrophobic initially, then become highly permeable to water and solute only after being triggered to release. The barrier, on the other hand, should be very hydrophilic, allowing water and solute to quickly diffuse across. This complicates integration of the two layers with a single sidewall material, as shown in Figure 10. Here the barrier and depot layers were cut to identical diameters $(15 \mathrm{~mm})$ and stacked in 5-pulse BMPR 


\begin{tabular}{|c|}
$139 \mu \mathrm{m}$ \\
\hline $143 \mu \mathrm{m}$ \\
$122 \mu \mathrm{m}$ \\
\hline $126 \mu \mathrm{m}$ \\
$126 \mu \mathrm{m}$ \\
\hline $127 \mu \mathrm{m}$ \\
\hline $130 \mu \mathrm{m}$ \\
\hline \\
\hline
\end{tabular}

\begin{tabular}{|c|}
\hline $141 \mu \mathrm{m}$ \\
\hline $142 \mu \mathrm{m}$ \\
$242 \mu \mathrm{m}$ \\
\hline $242 \mu \mathrm{m}$ \\
$248 \mu \mathrm{m}$ \\
\hline $24 \mathrm{\mu m}$ \\
\hline $248 \mu \mathrm{m}$ \\
\hline \\
\hline
\end{tabular}

Solute-free depot

PVA barrier

Depot

Impermeable wall

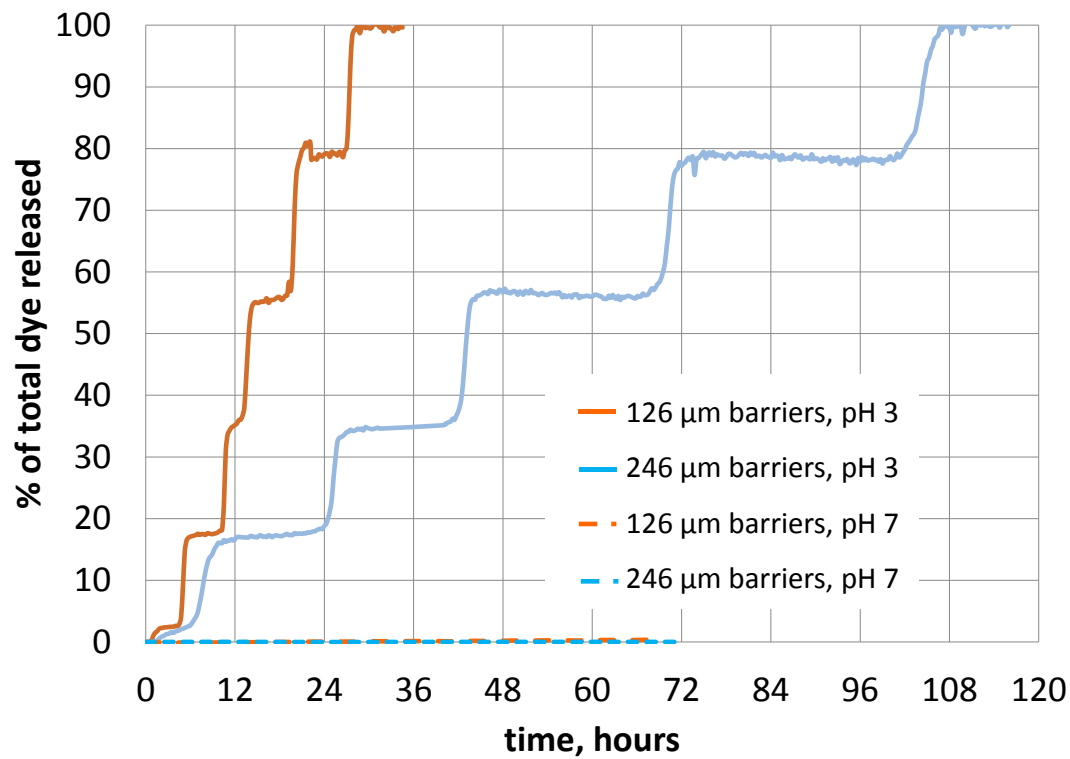

Figure 10: Configurations and release profiles for BMPR devices. (Left) Configurations of two BMPR devices. Solute-free depots are placed on top to guarantee initial adhesion to the impermeable wall. Note that devices are not drawn to scale; actual aspect ratio resembles a coin. (Right) Solute release profiles for the devices at $\mathrm{pH} 7$ (dotted) and $\mathrm{pH} 3$ (solid). No release observed at $\mathrm{pH}$ 7, while regular discrete pulses are observed at $\mathrm{pH} 3$.

devices. The devices had no discernable release during three days of immersion in $\mathrm{pH} 7$ buffer. At pH 3 we see sharp, discrete pulses at regular intervals corresponding to the barrier thickness. The release time for each pulse is less than one hour, as predicted for depot layers only $100 \mu \mathrm{m}$ thick. The lag time between pulses, however, is only $2 / 3$ as long as would be predicted for these barriers based on Equation 3. Pictures of the device, recorded every 15 minutes, show that the scavenger at the outer edge of each barrier layer is exhausted before the scavenger in the middle. Moreover, in a few instances the depot swelled and delaminated before the scavenger in the overlying barrier had even been consumed. Both problems point to poor contact between the hydrated barrier layer and the wax sidewall, allowing acid to permeate around the edge of the barrier and reach the depot layer earlier than planned. Despite the contact issues with these devices, however, control over the pulse periodicity by altering the barrier thickness is still 
conserved, with lag time between pulses increasing by a factor of four when the barrier thickness is doubled.

Barrier lag time can also be manipulated by altering the position of the scavenger within the barrier. As indicated in Equation 3, the lag time of the barrier varies linearly with the scavenger's center of mass: the effect of the scavenger is doubled when it is sequestered entirely at the downstream interface, while it is zero when all the scavenger is at the upstream interface and instantly consumed. Figure 11 shows devices constructed of identical films, with only the orientation of the barrier layers reversed. In these barriers, similar to the one shown via SEM in Figure $3 \mathrm{C}$, the $\mathrm{ZnO}$ is distributed evenly across the first $42 \%$ of the film thickness, with the remainder being $\mathrm{ZnO}$-free. These barrier layers were also cut $1 \mathrm{~mm}$ wider than the barriers used to demonstrate the contact issues in Figure 10, minimizing the opportunity for peripheral acid diffusion. When the $\mathrm{ZnO}$-laden side of the barriers faces the device exterior, the lag time is only $5.5( \pm 0.1)$ hrs, while when the orientation is reversed the lag time is $20.4( \pm 0.2)$ hrs, nearly

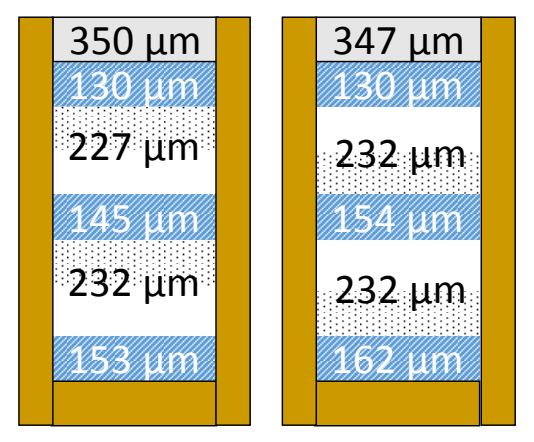

ZnO-free region in PVA bilayer ZnO-rich region in PVA bilayer Depot Impermeable wall Solute-free Depot



Figure 11: Effect of scavenger position on release profiles for BMPR devices. (Left) Configurations of two BMPR devices made from the same films, but with the orientation of the barrier layers reversed. Scavenger in barrier layer is restricted to one side (shaded region) of layer. Note that devices are not drawn to scale; actual aspect ratio resembles a coin. (Right) Solute release profiles for the devices at pH 7 and pH 3. Lag time can be strongly manipulated by controlling the location of the scavenger in the barriers. 
quadrupling the lag time without using any additional scavenger or increasing the barrier thickness. These lag times follow the relationship in Equation 3 within $3 \%$.

One important feature of BMPR systems over single reservoir systems is the ability to deliver different chemicals at different times from the same device. Figure 12 shows a device with alternating depots of methylene blue and methyl orange. Positively-charged methylene blue releases from the depot very quickly and can show discrete pulses even at high loadings and short lag times, whereas methyl orange is negatively charged and shows bimodal release, with comparatively rapid release while the cationic depot amine groups are saturated with solute, followed by slower release as the remaining solute molecules are displaced by other anions. This requires a longer minimum lag time between discrete pulses of methyl orange, but has no impact on the other solutes delivered during the interim. In this case, each solute was released once per

\begin{tabular}{|c|}
\hline $151 \mu \mathrm{m}$ \\
\hline $149 \mu \mathrm{m}$ \\
\hline $211 \mu \mathrm{m}$ \\
\hline $221 \mu \mathrm{m}$ \\
\hline $224 \mu \mathrm{m}$ \\
\hline $226 \mu \mathrm{m}$ \\
\hline $228 \mu \mathrm{m}$ \\
\hline \\
\hline \\
Solute-free depot \\
PVA barrier \\
Depot (Methylene Blue) \\
Depot (Methyl Orange) \\
Impermeable wall
\end{tabular}

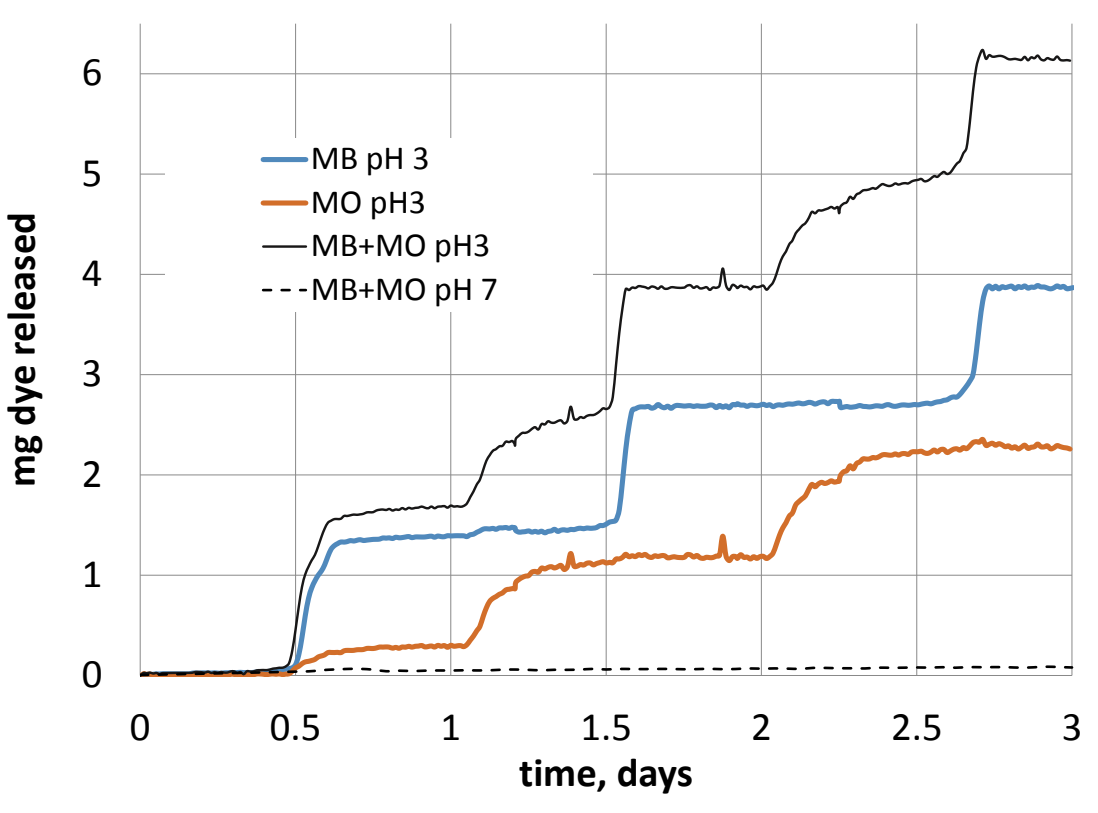

Figure 12: Release of multiple solutes from a BMPR device. (Left) Configuration of a BMPR device containing doses of both methylene blue and methyl orange. Note that devices are not drawn to scale; actual aspect ratio resembles a coin. (Right) Solute release profiles for the devices at $\mathrm{pH} 7$ and $\mathrm{pH} 3$. Solutes are released sequentially in 12 hour intervals. 
day, offset by 12 hours from each other, with lag times averaging $7 \%$ faster than the analytical predictions of Equation 3. Time-lapse videos of the release depicted in Figure 12 at $\mathrm{pH} 7$ and at pH 3 are included as Supplementary Information.

In theory, there is no hard limit on how many pulses can be delivered by a single device, as underscored by Figure 13, which shows ten pulses from one device. In practice, however, one can see that as the number of layers increases, the opportunity for the contact problems mentioned above also increases, with pulses 3-6 delaminating 30\% early. The rest of the pulses, and the last ones in particular, occurred within a few percent of their predicted time. A timelapse video of the release depicted in Figure 13 is included as Supplementary Information.

\section{Conclusions}

Currently, pulsatile delivery of solute requires either labor-intensive manual application
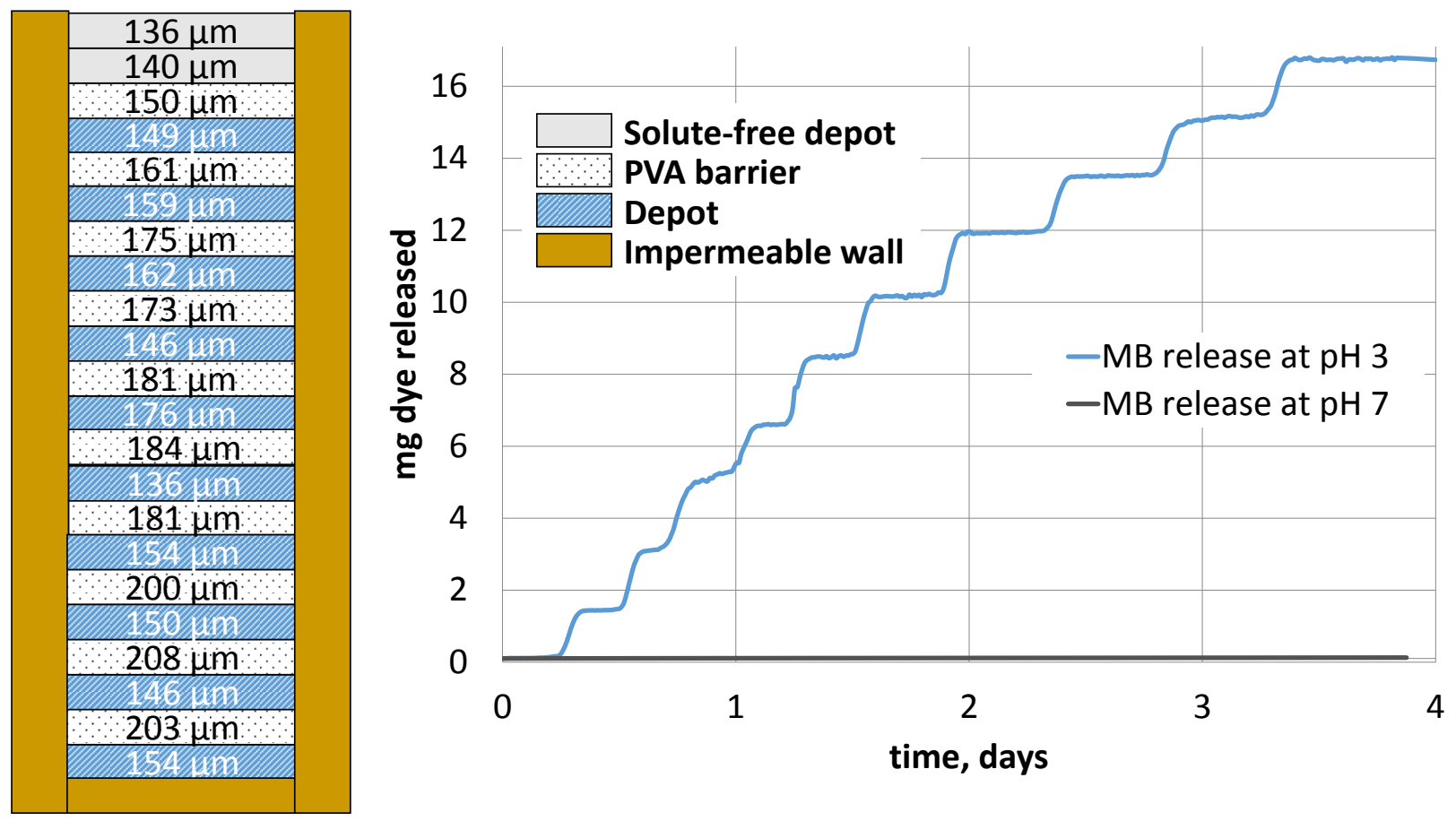

Figure 13: How many pulses can one BMPR device provide? (Left) Configuration of a 10-pulse BMPR device. Note that devices are not drawn to scale; actual aspect ratio resembles a pair of coins. (Right) Solute release profiles for the devices at $\mathrm{pH} 7$ and $\mathrm{pH} 3$. Most solutes, including the last four, released at their analytically predicted time, but the opportunity for wall-barrier contact problems increases with the number of doses. 
of every dose or expensive, complex devices. Barrier-mediated pulsatile release offers an elegant, materials-based alternative for pulsatile delivery. However, the linear dependence of erosion time on film thickness and the difficulty of maintaining perfect surface erosion across a wide range of thicknesses currently makes the use of polymer degradation as the timing mechanism for BMPR systems questionable. In this paper the BMPR approach has been decoupled from polymer degradation and instead linked to sacrificial scavenging of the external stimulant. This allows the delay time between pulses to be scaled across orders of magnitude with only modest changes in the barrier and opens the BMPR approach to a variety of external stimuli. PMMA/DMA hydrogels demonstrated complete immobilization of drug at $\mathrm{pH} 7$ yet

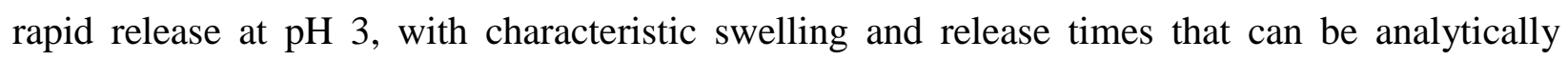
predicted and manipulated. The acid stimulant for these depots can be blocked by ZnO-loaded polymer hydrogels, preventing solute release for a prescribed period of time, followed by rapid breakthrough of stimulant and release of solute. This delay time can be manipulated by the barrier thickness, its scavenger concentration and its scavenger location, with little hindrance of the stimulant afterwards. The number of pulses incorporated into a single device is limited only by the art of making them, with devices releasing up to 10 pulses demonstrated here. Importantly, different chemicals can be incorporated into each pulse, allowing automatic sequential delivery of multiple solutes.

This raises the spectre of inexpensive, customized delivery systems, where off-the-shelf layers are stacked in a particular configuration at the point of use and sealed into a simple polymer chip. There are no electronic or mechanical components, no power supply, and no circuitry, greatly reducing both the complexity and volume of the system, as well as its cost. The stimulant for the demonstrated system, citric acid, could be easily replaced by other organic 
acids, potentially derived from other organic molecules enzymatically; gluconic acid derived from blood sugar being one example.

\section{Acknowledgements}

The authors gratefully acknowledge the University of Iowa, the National Science Foundation (CBET-1133297), and the American Heart Association (11SDG7600044) for their support of this work. Y.N. participated in this work through a travel grant from the Institute of National Colleges of Technology, Japan (2014-146). 


\section{References}

[1] G. Brabant, K. Prank, C. Schöfl, Pulsatile Patterns in Hormone Secretion, Trends in Endocrinology and Metabolism, 3 (1992) 183-190.

[2] Centers for Disease Control and Prevention, Rabies Vaccine: What You Need to Know, www.cdc.gov/vaccines/pubs/vis/downloads/vis-rabies.pdf, (2009).

[3] R.A. Siegel, C.G. Pitt, A strategy for oscillatory drug release. General scheme and simplified theory, Journal of Controlled Release, 33 (1995) 173-188.

[4] J.T. Li, R.F. Lockey, I.L. Bernstein, J.M. Portnoy, R.A. Nicklas, Allergen Immunotherapy: a practice parameter, Annals of Allergy, Asthma \& Immunology, 90 (2003) 1-40.

[5] J. Abrams, A reappraisal of nitrate therapy, Journal of the American Medical Association, 259 (1988) 396-401.

[6] W.A. McKim, Drugs and Behavior. An Introduction to Behavioral Pharmacology, 2007.

[7] M. McCoy, Microencapsulation aids insecticides, Chemical and Engineering News, 87 (2009) 16.

[8] A. Gazzaniga, L. Palugan, A. Foppoli, M.E. Sangalli, Oral pulsatile delivery systems based on swellable hydrophilic polymers, European Journal of Pharmaceutics and Biopharmaceutics, 68 (2008) 11-18.

[9] E.S.K. Tang, L.W. Chan, P.W.S. Heng, Coating of multiparticulates for sustained release, American Journal of Drug Delivery, 3 (2005) 17-28.

[10] K. Fujioka, S. Sato, Y. Takada, Y. Sasaki, N. Tamura, Sustained pulsewise release pharmaceutical preparation, European Patent no. 230654 (1987).

[11] H.L. Jiang, K.J. Zhu, Pulsatile protein release from a laminated device comprising of polyanhydrides and pH-sensitive complexes, International Journal of Pharmaceutics, 194 (2000) 51-60.

[12] X. Liu, G.J. Pettway, L.K. McCauley, P.X. Ma, Pulsatile release of parathyroid hormone from an implantable delivery system, Biomaterials, 28 (2007) 4124-4131.

[13] J.H. Jeon, D.A. Puleo, Alternating release of different bioactive molecules from a complexation polymer system, Biomaterials, 29 (2008) 3591-3598.

[14] J. Siepmann, A. Göpferich, Mathematical modeling of bioerodable, polymeric drug delivery systems, Advanced Drug Delivery Reviews, 48 (2001) 229-247.

[15] F. von Burkersroda, L. Schedl, A. Göpferich, Why degradable polymers undergo surface erosion or bulk erosion, Biomaterials, 23 (2002) 4221-4231.

[16] T. Tanaka, D. Fillmore, S.-T. Sun, I. Nishio, G. Swislow, A. Shah, Phase Transitions in lonic Gels, Physical Review Letters, 45 (1980) 1636-1639.

[17] R.A. Siegel, B.A. Firestone, pH-dependent equilibrium swelling properties of hydrophobic polyelectrolyte copolymer gels, Macromolecules, 21 (1988) 3254-3259.

[18] K. Kataoka, H. Miyazaki, M. Bunya, T. Okano, Y. Sakurai, Totally Synthetic Polymer Gels Responding to External Glucose Concentration: Their Preparation and Application to On-Off Regulation of Insulin Release, Journal of the American Chemical Society, 120 (1998) 12694-12695.

[19] K. Sumaru, M. Kameda, T. Kanamori, T. Shinbo, Characteristic Phase Transition of Aqueous Solution of Poly(N-isopropylacrylamide) Functionalized with Spirobenzopyran, Macromolecules, 37 (2004) 4949-4955. 
[20] J. Lipták, J. Nedbal, M. Ilavsky, Dielectric behaviour of swollen poly(acrylamide)gels in the collapse region, Polymer Bulletin, 18 (1987) 81-86.

[21] T. Miyata, N. Asami, T. Uragami, A reversibly antigen-responsive hydrogel, Nature, 399 (1999) 766 769.

[22] B.A. Firestone, R.A. Siegel, Dynamic pH-dependent swelling properties of a hydrophobic polyelectrolyte gel, Polymer Communications, 29 (1988) 204-208.

[23] R.A. Siegel, M. Falamarzian, B.A. Firestone, B.C. Moxley, pH-controlled release from hydrophobic/polyelectrolyte copolymer hydrogels, Journal of Controlled Release, 8 (1988) 179-182.

[24] S.S. Halacheva, D.J. Adlam, E.K. Hendow, T.J. Freemont, J. Hoyland, B.R. Saunders, Injectable biocompatible and biodegradable $\mathrm{pH}$-responsive hollow particle gels containing poly(acrylic acid): the effect of copolymer composition on gel properties, Biomacromolecules, 15 (2014) 1814-1827.

[25] E.E. Nuxoll, E.L. Cussler, The third parameter in reactive barrier films, AIChE Journal, 51 (2005) 456463.

[26] E.E. Nuxoll, R.A. Siegel, E.L. Cussler, Layered reactive barrier films, Journal of Membrane Science, 252 (2005) 29-36.

[27] R.A. Siegel, I. Johannes, C.A. Hunt, B.A. Firestone, Buffer Effects on Swelling Kinetics in Polybasic Gels, Pharmaceutical Research, 9 (1992) 76-81.

[28] B.A. Firestone, R.A. Siegel, Kinetics and Mechanisms of Water Sorption in Hydrophobic, Ionizable Copolymer Gels, Journal of Applied Polymer Science, 43 (1991) 901-914.

[29] D.G. Leaist, The effects of aggregation, counterion-binding, and added $\mathrm{NaCl}$ on diffusion of aqueous methylene blue, Canadian Journal of Chemistry, 66 (1988) 2452-2457.

[30] N.K. Das, B.M. Mandal, Methylene Blue as a Retarder of Free Radical Polymerization: 1. Polymerization of Acrylonitrile, Methyl methacrylate, and Styrene, Polymer, 23 (1982) 1653-1658.

[31] K.F. Finger, A.P. Lemberger, T. Higuchi, L.W. Busse, D.E. Wurster, Investigation and Development of Protective Ointments IV, Journal of the American Pharmaceutical Association, 49 (1960) 569-573.

[32] W.I. Higuchi, T. Higuchi, Theoretical Analysis of Diffusional Movement Through Heterogeneous Barriers, Journal of the American Pharmaceutical Association, 49 (1960) 598-606.

[33] R.A. Siegel, E.L. Cussler, Reactive Barrier Membranes: some theoretical observations regarding the time lag and breakthrough curves, Journal of Membrane Science, 229 (2004) 33-41. 
